\title{
False Positive Meta-Iodobenzyl-Guanidine Scan in Testis Dermoid Cyst: A Case Report
}

\author{
Hamed Akhavizadegan ${ }^{1,}$ \\ ${ }^{1}$ Assistant Professor in Urology, Baharloo Hospital, Tehran University of Medical Sciences, Tehran, IR Iran \\ "Corresponding author: Hamed Akhavizadegan, Urology Department, Baharloo Hospital, Behdari Square, RahAhan Square, Tehran, IR Iran. Tel: +98-2155658501, Fax: \\ +98-2155648189, E-mail: h-akhavizadegan@tums.ac.ir \\ Received 2017 March 06; Accepted 2017 April 05.
}

\begin{abstract}
Introduction: This study aimed at presenting a case of testis dermoid tumor with false positive meta-iodobenzyl-guanidine (MIBG) scan.

Case Report: A 16-year-old male was referred to the urology service with bilateral testicular tumor. Testis carcinoid tumor was diagnosed after right radical orchiectomy. Although the MIBG scan was positive in left hemiscrotum before left sided operation, the left side pathology reported testis dermoid cyst.

Conclusions: Meta-Iodobenzyl-Guanidine can be absorbed by adipose tissue, one of the components of dermoid cysts. This phenomenon can result in false positivity of this scan in these cases. False negative MIBG scan is not uncommon in carcinoid tumors, however, false positive answers should not be neglected in planning treatment.
\end{abstract}

Keywords: Testis Tumor, Dermoid, Meta-Iodobenzyl-Guanidine, Nuclear Scan

\section{Introduction}

Carcinoid tumor of the testis accounts for less than $1 \%$ of all testicular neoplasms. Discrimination between primary or secondary types of the tumor and metastasis detection is very important in the management of the disease (1). Two types of nuclear scintigraphy have been recommended for this purpose, the first and more specific, somatostatin receptor scan, and the second and less specific, meta-iodobenzyl-guanidine (MIBG) scan $(1,2)$. MetaIodobenzyl-guanidine (MIBG) scan has been reported to have high rates of false negative results. Nevertheless, reports on false positive MIBG scan in testis dermoid tumor have not been previously reported. This report aimed at presenting an unusual case of testicular dermoid tumor with false positive MIBG scan.

\section{Case Presentation}

A 16-year-old male was admitted to the urology clinic, complaining of right testicular pain for one week. He had a history of trauma to the right testis leading to ipsilateral pain and hematoma nearly 3 months before referral. At the time of current presentation, the testis size was not reduced to its normal size.

On examination, he had a hard and large mass instead of right testis and a small, firm, and well-defined mass in the upper border of the left testis, markedly suggestive of bilateral testis tumor.
Ultrasound examination showed a mixed echogenic mass occupying the majority of the right testis measuring $45 \times 47 \times 31 \mathrm{~mm}$ with $4 \mathrm{~mm}$ calcification and an isoechoic mass; $14 \times 24 \mathrm{~mm}$ for the upper part of the left testis.

In order to diagnose any possible metastasis, chest $\mathrm{X}$ ray and testis tumor markers (lactate dehydrogenase, beta human chorionic gonadotropin, alpha-fetoprotein) were used, all of which appeared to be normal. Right radical orchidectomy was subsequently performed.

The testis was entirely replaced by the tumor. It was decided to wait for the right testis tumor pathology and to arrange sperm freezing before any procedures for the left testis tumor.

On macroscopic view, there was a $4.5-\mathrm{cm}$ well-defined creamy mass lesion with solid and firm lobulated cream cut sections bearing hemorrhagic and necrotic foci.

Pathology review showed a well demarcated tumor composed of monomorphic cells hosted in a nested trabecular manner. The neoplastic cells had granular chromatin and rarely with mitotic features. Immunohistochemistry survey showed positive staining with, Pan-CK, synaptophysin, and chromogranin.

No Calretinin or Vimentin were detected in the tumor. Proliferative activity (Ki67) was not significant. These features were compatible with a pure well-differentiated carcinoid tumor. The tumor was confined to testis capsule without any lymphovascular invasion.

Chest, abdomen and pelvis CT scan revealed no

Copyright ( $\odot$ 2017, Nephrology and Urology Research Center. This is an open-access article distributed under the terms of the Creative Commons 
significant finding regarding para-aortic and iliac lymphadenopathy or pulmonary abnormality.

Complete gastroenterology work up was performed to rule out the possibility of testicular metastasis from an extra-testicular carcinoid tumor and a sample of the patient's semen was frozen.

In order to verify the pathology in the contralateral testis, it was decided to use nuclear scan. Because of unavailability of nuclear octreotide scan, a MIBG (metaiodobenzyl-guanidine) scan was requested, which was positive on the left hemiscrotum.

With diagnosis of bilateral primary testis carcinoid tumor, the patient was admitted for left partial orchiectomy. Partial orchiectomy was performed classically and a pale, creamy white, firm mass with sharp borders was excised from left testis after separating the head of the epididymis. Surprisingly, the pathologist reported dermoid cyst without any carcinoid content.

After 5 years of follow-up, the patient was good with acceptable spermogram. No tumor marker, including urinary 5-hydroxyindoleacetic acid (5-HIAA) or imaging was positive although these tests are not considered to be very specific (2).

\section{Discussion}

This is the first case report of testicular carcinoid tumor concomitant with contralateral testis dermoid cyst. Testicular carcinoid is a rare testicular neoplasm. There are 2 different types of carcinoid testis tumors, including primary carcinoid tumors without the involvement of any other organ and secondary carcinoid testis tumor as a metastasis from other source (2).

The most important part of diagnostic management of this disease is diagnosis of primary from secondary tumors. In addition to general imaging and endoscopic workups, nuclear scan has a key role in this discrimination (1).

One of the diagnostic tools for carcinoid tumors is somatostatin receptor scintigraphy (SRS). Somatostatin receptors are present in $70 \%$ to $90 \%$ of carcinoid tumors, so this scan is positive in the same percentage of afflicted patients (1).

According to the literature, somatostatin receptor scintigraphy diagnoses carcinoid tumor with higher sensitivity (more than 50\%) when compared with MIBG scintigraphy. This means that if we use MIBG scintigraphy to diagnose Carcinoid tumor, it is expected that $50 \%$ of true cases would be negative on the test (2).

Some other tissue types such as adipose tissue can absorb MIBG, as well. Thus, several cases have been reported with false positive results of MIBG in angiomyolipoma and adrenocortical carcinoma (3).

This case report presented a patient with primary carcinoid tumor in right testis and dermoid cyst on the left side.

To the best of our knowledge, no false positive MIBG scan has been reported in dermoid cysts. Dermoid cyst is a type of mature teratoma and can have adipose contents and also it is not rare to see dermoid cyst as a component of carcinoid tumor (4).

Because adipose tissue can absorb MIBG, in our case a false positive result occurred in the presence of dermoid cyst in another testis.

Dermoid cyst is among benign testis tumors with good prognosis and only needs partial orchiectomy. It has been seen commonly with carcinoid testis tumor, which has resulted a separate group of carcinoid tumors named carcinoid tumors occurring as part of teratomas. The case confronted the possibility of presentation of carcinoid as a part of teratoma as carcinoid testis and teratoma in different testes.

\subsection{Conclusions}

Among other false positive cases of MIBG scan, dermoid cyst of testis should be included. Although false negative scan is very important, false positivity of it can cause unnecessary interventions.

\section{References}

1. Glazier DB, Murphy DP, Barnard N, Cummings KB, Weiss RE. Primary carcinoid tumour of the testis. BJU Int. 1999;83(1):153-4. [PubMed: 10233475].

2. Neely D, Gray S. Primary carcinoid tumour of the testis. Ulster Med J 2011;80(2):79-81. [PubMed: 22347748].

3. Hadi M, Chen CC, Whatley M, Pacak K, Carrasquillo JA. Brown fat imaging with (18)F-6-fluorodopamine PET/CT, (18)F-FDG PET/CT, and (123)IMIBG SPECT: a study of patients being evaluated for pheochromocytoma. J Nucl Med. 2007;48(7):1077-83. doi: 10.2967/jnumed.106.035915. [PubMed: 17574980].

4. Garrido Abad P, Herranz Fernandez LM, Jimenez Galvez M, Suarez Fonseca C, Santos Arrontes D, Nieto Llanos S, et al. [Mature cystic teratoma of the testis (dermoid cyst). Case report and literature review]. Arch Esp Urol. 2009;62(9):747-51. [PubMed:19955600]. 